\title{
SUPERNOVA TYPES AND RATES *
}

\author{
Enrico Cappellaro \\ cappellaro@pd.astro.it \\ Massimo Turatto \\ turatto@pd.astro.it \\ Osservatorio Astronomico di Padova \\ vicolo dell'Osservatorio, 5 \\ I-35122, Padova (Italy)
}

\begin{abstract}
We review the basic properties of the different supernova types identified in the current taxonomy, with emphasis on the more recent developments. To help orienting in the variegate zoo, the optical photometric and spectroscopic properties of the different supernova types are presented in a number of summary figures.

We also report the latest estimates of the supernova rates and stress the need for a dedicated effort to measure SN rates at high redshift.
\end{abstract}

\section{INTRODUCTION}

Supernovae $(\mathrm{SNe})$ are at the confluence of many different streams of astronomical researches. As the final episode of the life of many kinds of stars, SNe allow crucial tests of stellar evolution theories. Yet, in many cases we are still entangled with the basic problem of finding a viable progenitor scenario for each SN type. During and immediately after the explosions, there are a variety of fundamental physical mechanisms which can be probed, such as neutrino and gravitational wave emissions, flame propagation and explosive nucleosynthesis, radioactive decays and shocks with circumstellar matter. Relics of the explosions are collapsed remnants, neutron stars or black holes, and expanding gas clouds which

\footnotetext{
* Most of the material presented in this paper has been retrieved from the Padova Archive of SN observations. This is the product of a long standing effort for the monitoring of $\mathrm{SNe}$ conducted using ESO-La Silla and Asiago telescopes (Turatto et al. 1990).
} 


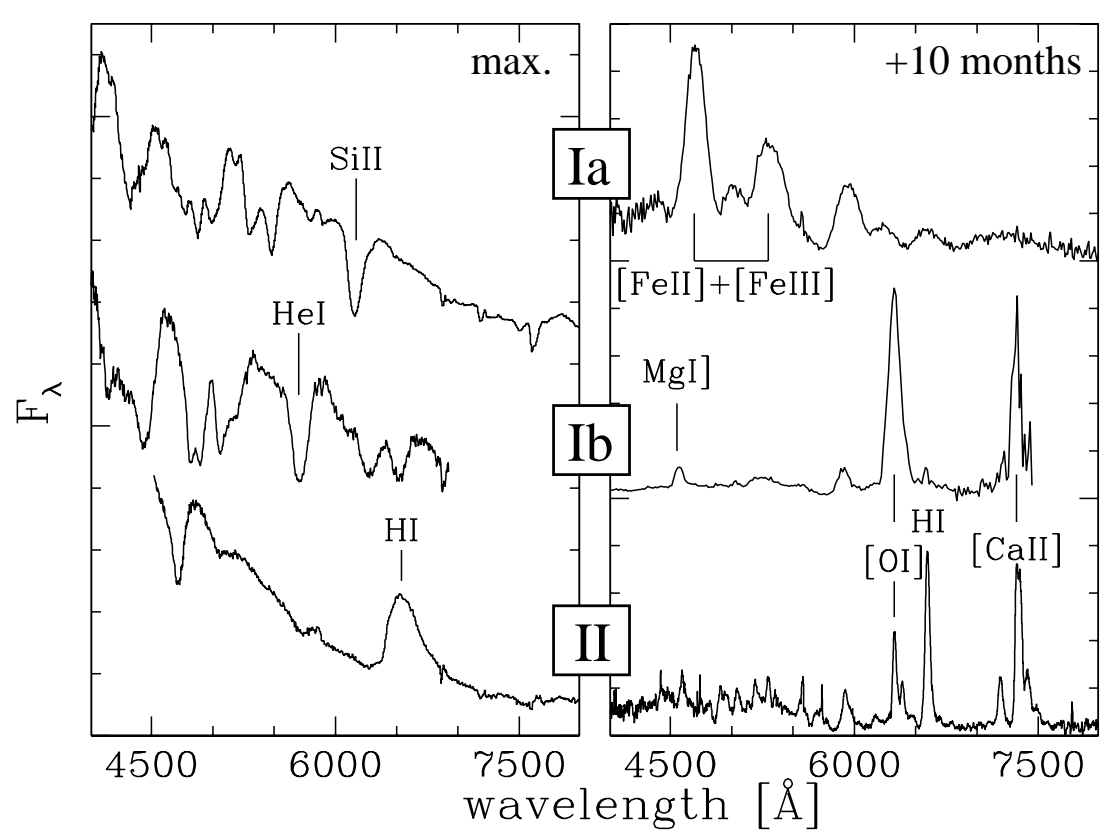

Figure 1. Basic SN types spectra. A SN which near maximum (left panel) shows clear signature of $\mathrm{H} \alpha$ is defined as type II, if it shows a strong SiII absorption at about $6150 \AA$ is a type Ia, otherwise it is of type Ib/c (in the figure we show a SNIb characterized by strong He lines). Ten months later (right panel) SN Ia show strong emissions of [FeII] and [FeIII], SN Ib/c are dominated by [CaII] and [OI]. These same lines and strong $\mathrm{H} \alpha$ emission are typical of SNII.

heat and pollute the interstellar medium. Indeed, SNe are a main agent in the chemical evolution of galaxies.

Moreover, because of their huge luminosity and the fact that they can be accurately calibrated, type Ia $\mathrm{SNe}$ are the best distance indicators for measuring the geometry of the Universe.

For all these reasons, in the last decades there has been an increased interest in SN studies and a renewed effort in SN searches. As a consequence, the number of SN discoveries has increased from about 20/yr in the early ' 80 , to about 200/yr in the last few years raising the number of SNe discovered to date to $\sim 1800$ (Barbon et al. 1999;

http://merlino.pd.astro.it/ supern/ for a frequently updated version). It is not surprising that the enlarged sample combined with the better quality of the observations obtained by the new generation instruments is deeply changing our understanding of the SN phenomenon. 
The aim of this paper is to give a snapshot of the SN zoo including the latest acquisitions. Other information can be found in Filippenko (1997), Wheeler and Benetti (2000) and reference therein. We will also present the latest estimates of the SN rates which are fundamental to link the evolution of stars and stellar systems.

\section{BASIC SN TYPES}

The affair of SN classification began with Minkowskii (1941) who noticed that there are at least two different kinds of SNe, showing (type II) and not showing (type I) $\mathrm{H}$ in their spectra.

This subdivision was maintained until, in the ' 80 s, it was recognized that a number of peculiar SN I missing in the spectrum near maximum the typical feature at $\sim 6510 \AA$, had a completely different nebular spectrum dominated by forbidden $\mathrm{Ca}$ and $\mathrm{O}$ emissions instead of $\mathrm{Fe}$ lines (Gaskell et al. 1986). Examining more carefully the near maximum spectra it was noticed that these SNe come in at least two flavors: those showing strong He lines were labeled Ib, the others Ic. We will argue later however that this subdivision is at a lower hierarchical level. The first branch of the SN classification is illustrated in Fig. 1.

In general, in the early phases the optical depth of the ejecta remains high and the emergent spectrum only probes the outermost layers. Therefore the early spectrum is most sensitive to variations in the density and composition of the progenitor envelope. With time the density and temperature decrease, the photosphere recedes in mass coordinates and eventually the ejecta becomes optically thin. At this point the innermost regions becomes exposed and the yields of the explosions can be probed. The fact that the nebular spectrum of $\mathrm{SN} \mathrm{Ib/c} \mathrm{is} \mathrm{similar} \mathrm{to}$ that of SN II, but for the $\mathrm{H}$ emission, prompts for a similar explosion mechanism. Instead, the weakness of intermediate element emissions, in particular $\mathrm{O}$, in the nebular spectrum of $\mathrm{SN}$ Ia excludes that the progenitors of SN Ia are massive stars.

The SN taxonomy which originally was based on spectra near maximum may result somewhat confusing when one try to build a coherent progenitor scenario: indeed a more physical classification could be based on the distinction between $\mathrm{SNe}$ arising from the collapse of massive stars (SNII and SNIb/c) and SNe due to thermonuclear explosions of low mass stars (SN Ia). In the following we will give a brief description of the characteristics of each SN type describing the detailed taxonomy illustrated in Fig. 2. 


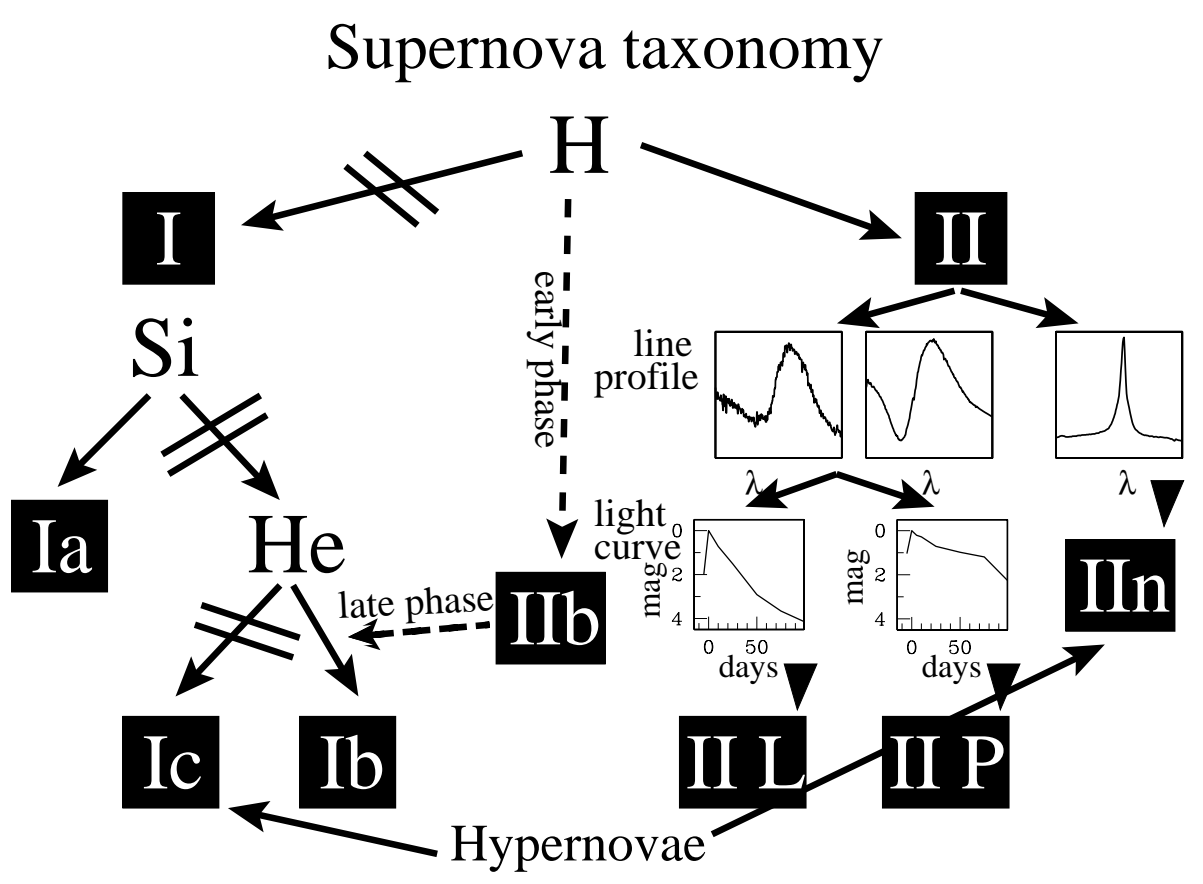

Figure 2. The detailed classification of SNe requires not only the identification of specific features in the early spectra, but also the analysis of the line profiles, luminosity and spectral evolutions

\section{TYPE IA SNE}

SN Ia are quite homogeneous events with similar luminosity and spectral evolution. Indeed until the early '90s, it was commonly accepted that SN Ia were identical and that observed differences were mainly due to observational errors (e.g Leibundgut \& Tammann 1990). With the improvement of the signal to noise of the observations it was definitely demonstrated that differences do exist. In particular, though the light curve shapes remain similar, the absolute luminosities and the decline rates are different (Fig. 3). Actually, a relation has been found between the rate of the luminosity decline and the absolute magnitudes at maximum, with fast declining SNe being fainter (and redder). Because this is crucial for recovering SN Ia as distance indicators, a large efforts is being devoted to the accurate calibration of the different expressions of this relation (Phillips et al. 1999, Riess et al. 1998, Perlmutter et al. 1999). To some extent, the spread in luminosity correlates with variations in the spectral properties (Nugent et al. 1995). As shown in Fig. 4, very 


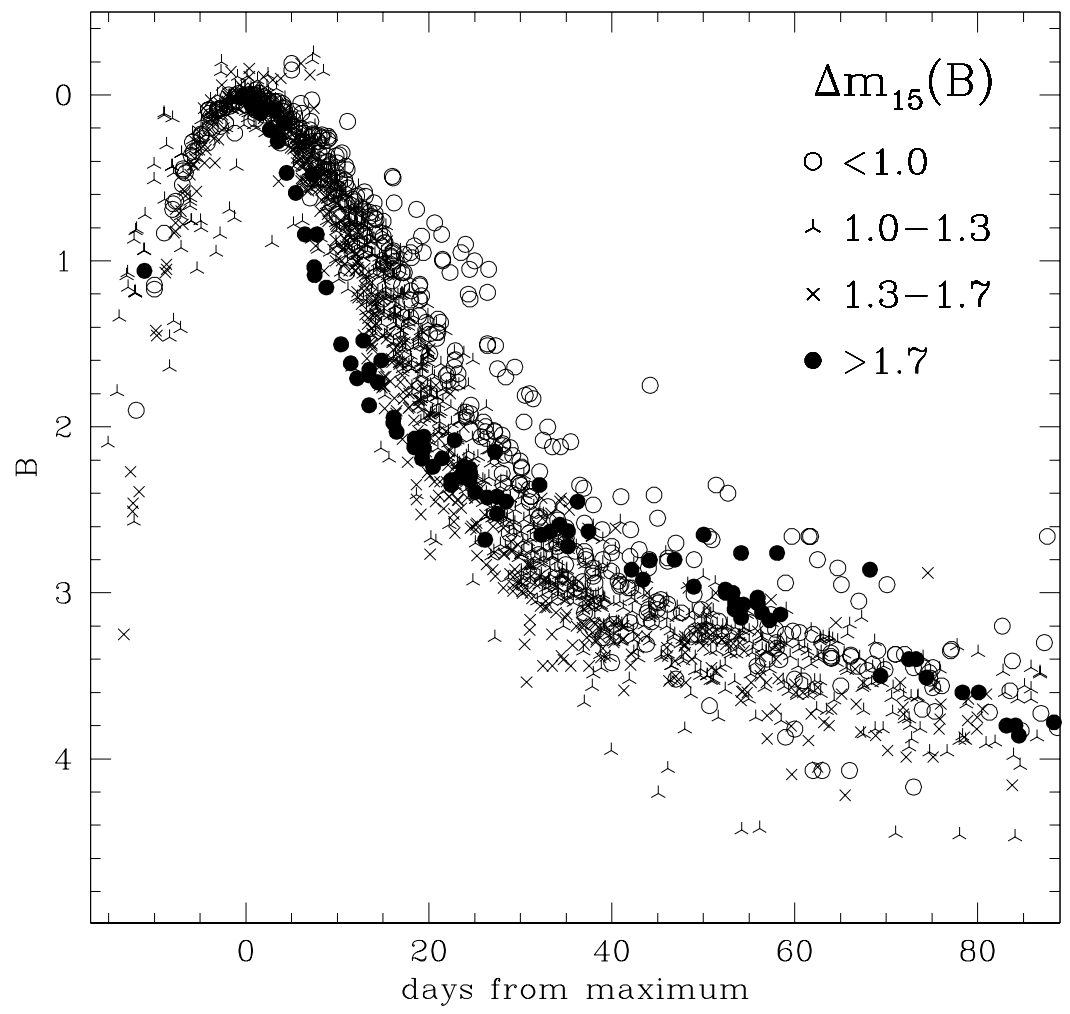

Figure 3. Light curves of $87 \mathrm{SN}$ Ia relative to the phase and magnitude of maximum. The symbols identify objects with different $\Delta m_{15}(B)$, the magnitude decline in the early 15 days (Altavilla 2000).

bright or very faint SNe show distinctive spectral features, whereas the differences among "normal" SN Ia stand out only after a careful analysis.

It is generally agreed that the difference in luminosity call for one order of magnitude variance in the mass of the radioactive ${ }^{56} \mathrm{Ni}$ which is powering the early light curve. Controversial is the claim that light curves and spectral synthesis models require a factor two in the total progenitor mass (Cappellaro et al. 1997). The standard scenario for the SN Ia explosion consists of a binary system where one of the star is a white dwarf (WD) which accretes matter for the secondary star, reaches the Chandrasekhar limit, and undergoes a disruptive, thermonuclear explosion (Nomoto et al. 1984, Woosley \& Weaver 1986). It is still unclear whether it is possible to reproduce the observed variance of SN Ia within the standard scenario (Mazzali et al. 2000). 


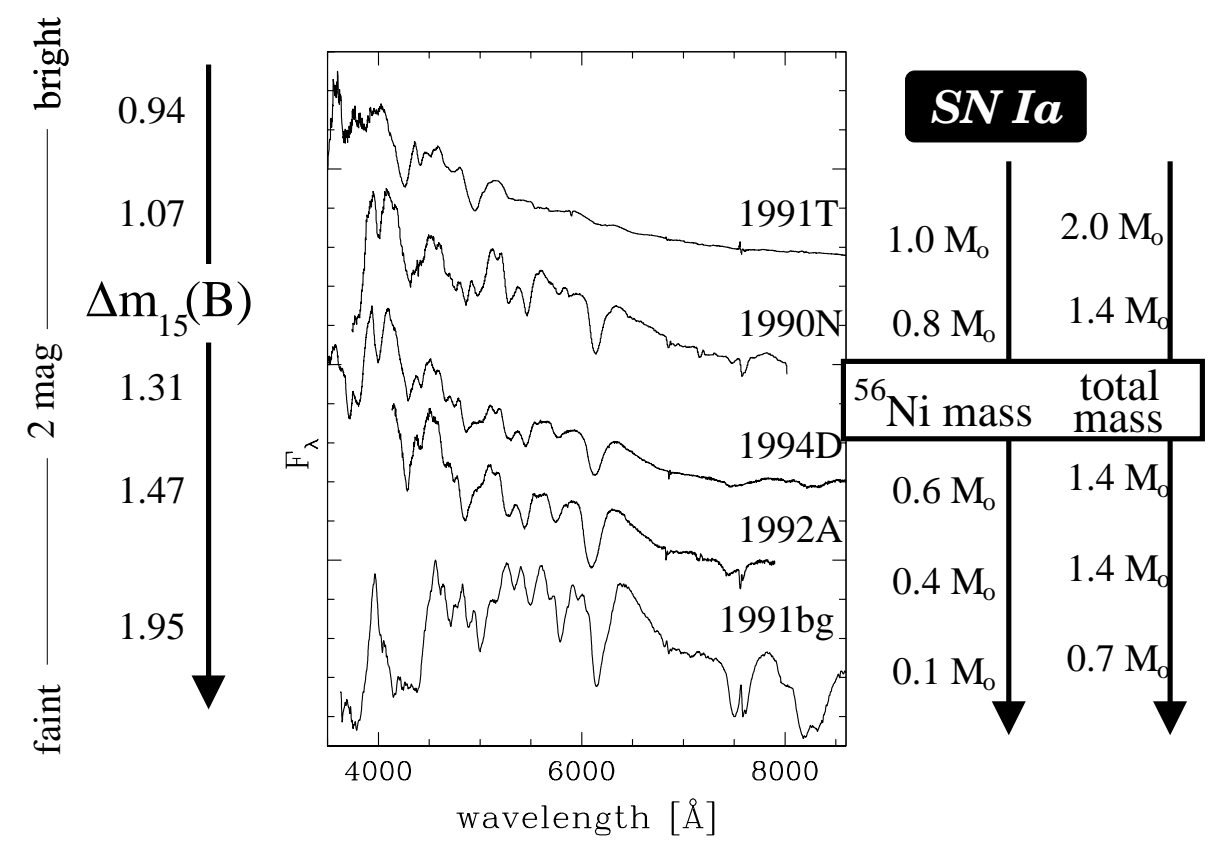

Figure 4. Comparison of the maximum light spectra of SN Ia with different decline rates (left), hence luminosities. On the right the ranges in ${ }^{56} \mathrm{Ni}$ and total masses suggested by light curve and spectral synthesis models are indicated.

An interesting finding in this respect is that on average SN Ia in early type galaxies show a faster decline rate (hence are less luminous) than SN Ia in late type galaxies (Fig. 5, cf. van den Bergh \& Pazder(1992), Hamuy et al.(2000)). This suggests that (some of) the variance of SN Ia is related to different ages of the progenitor population. Until this is not well understood, a severe caveat remains on the use of SN Ia as cosmological distance indicators.

\section{TYPE II SNE}

One might say that the only thing in common for SN II is the presence of $\mathrm{H}$ lines in the spectra. Indeed the maximum luminosities range over two orders of magnitudes and the spectral energy distributions and line profiles also shows dramatic differences. Historically, the first attempt to sort out some order was based on the light curve shapes (Barbon et al. 1979). In some cases, SN II after maximum remain on a plateau of almost constant luminosity for periods up to 2-3 months (II-P), in 


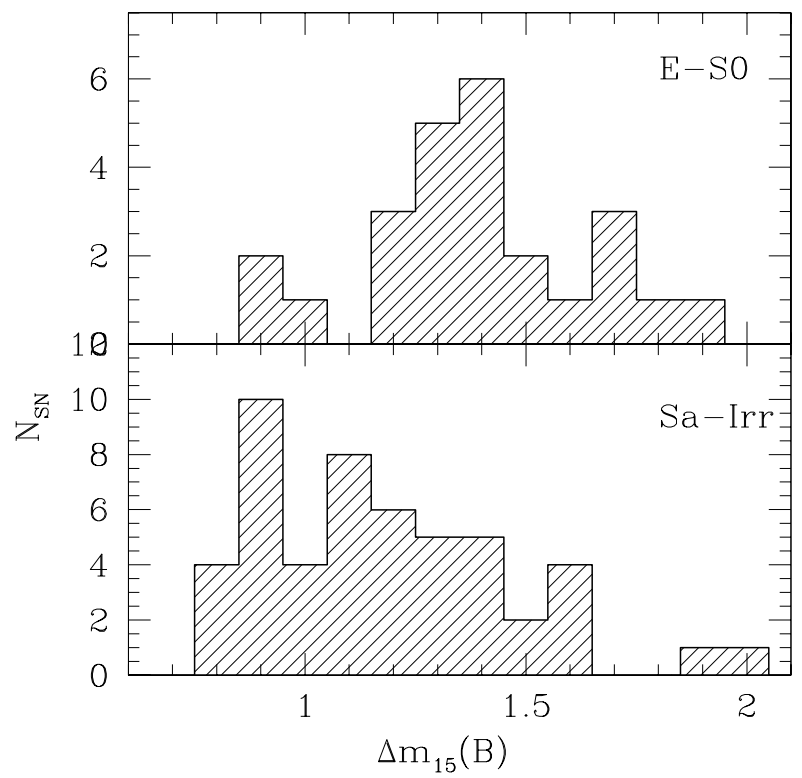

Figure 5. Histogram of $\Delta m_{15}(B)$ for SN Ia in early type galaxies (upper panel) and in late type galaxies (lower panel). A Kolmogorov-Smirnov test shows that the probability that the two distributions derive from the same population is only 0.004 (Altavilla 2000).

other cases they decline more or less linearly (II-L). The two classes are not separated and intermediate cases do exist (Clocchiatti et al. 1996). An extreme case of SN II-P is usually considered SN 1987A, the best observed SN ever, which after maximum showed a steadily increase of magnitude, lasting abound 3 months, until eventually the decline began (Fig. 6).

In general, it is assumed that SNII-L are brighter than SN IIP, but there are many exceptions (Patat et al. 1994), e.g. SN 1983K the bright II- P shown in Fig. 6. At late time $(>200 \mathrm{~d})$, many SN II settle on a linear decline rate of about $1 \mathrm{mag} / 100 \mathrm{~d}$. This is powered by the second branch of the radioactive decay chain ${ }^{56} \mathrm{Ni}-{ }^{56} \mathrm{Co}-{ }^{56} \mathrm{Fe}$. The fact that, despite the enormous difference at maximum, SN II at late time converge to a similar luminosity was taken as an indication that the ${ }^{56} \mathrm{Ni}$ mass produced in SN II was very similar, namely $\sim 0.1 \mathrm{M}_{\odot}$ (Turatto et al. 1990). Actually, even in this respect there may be significant variations ( Schmidt et al. 1994). An extreme case is that of SN 1997D (Fig. 6) which produced only $0.002 \mathrm{M}_{\odot}$ of ${ }^{56} \mathrm{Ni}$ and showed extremely low expansion velocities (Turatto et al. 1998). 


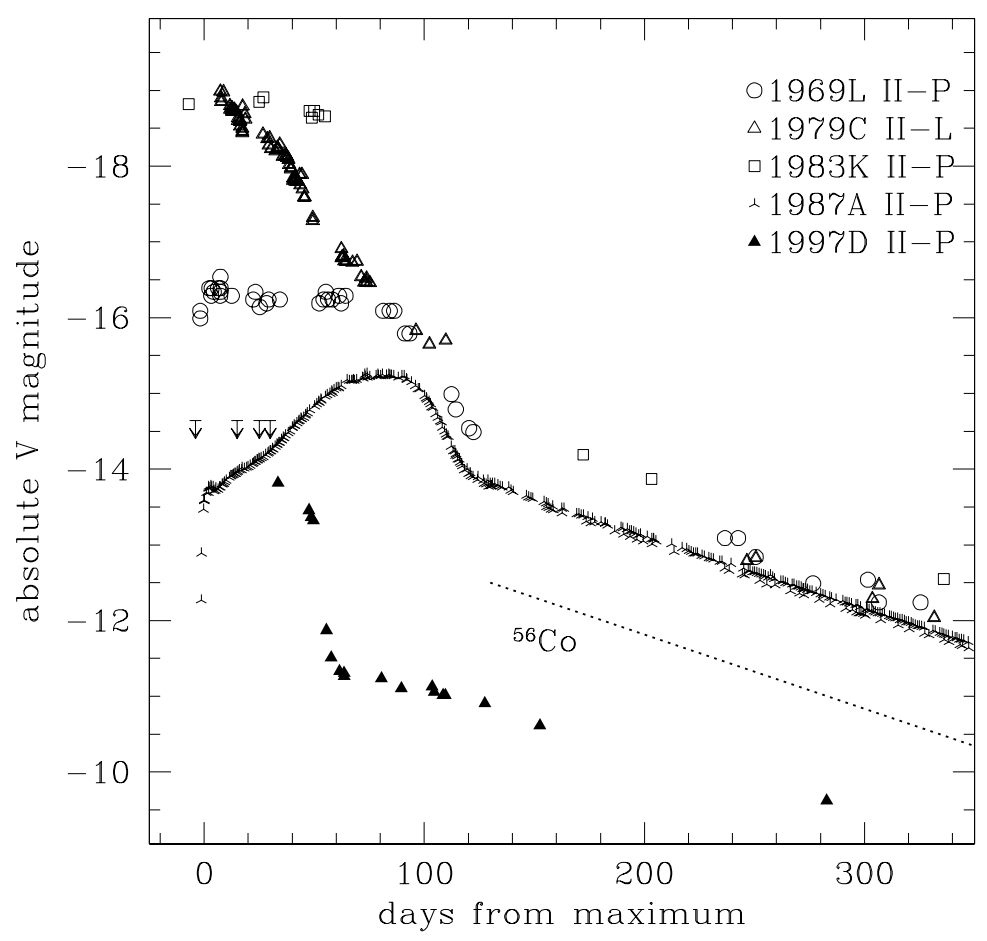

Figure 6. Representative light curves of SN II. The dotted line is the expected luminosity decline rate when the light curve is powered by the decay of ${ }^{56} \mathrm{Co}$.

It is generally accepted that SN II result from the core collapse of stars in the range of $10-30 \mathrm{M}_{\odot}$ (Woosley et al. 1988). Because of various mechanisms (eg. difference in metallicities, interaction in binary system) the size and the mass of the $\mathrm{H}$ envelope at the time of explosion can be very different even for progenitors of the same initial mass. In a typical SN IIP the $\mathrm{H}$ envelope mass is $\sim 5-10 \mathrm{M}_{\odot}$ and the radius is $\sim 10^{15} \mathrm{~cm}$. The shock wave originated by the explosion rapidly reaches the surface completely ionizing the $\mathrm{H}$. When the ejecta expands the energy from the recombination of $\mathrm{H}$ sustains the photosphere at an almost constant radius and temperature. The length of the plateau depends on the envelope mass and when this gets very small $\left(<1-2 \mathrm{M}_{\odot}\right)$ the decline is faster (SN IIL). The different line profiles of II-P and II-L, with II-L missing the P-Cygni absorption of the Balmer lines (Fig. 7) is also due to their reduced envelope masses. 


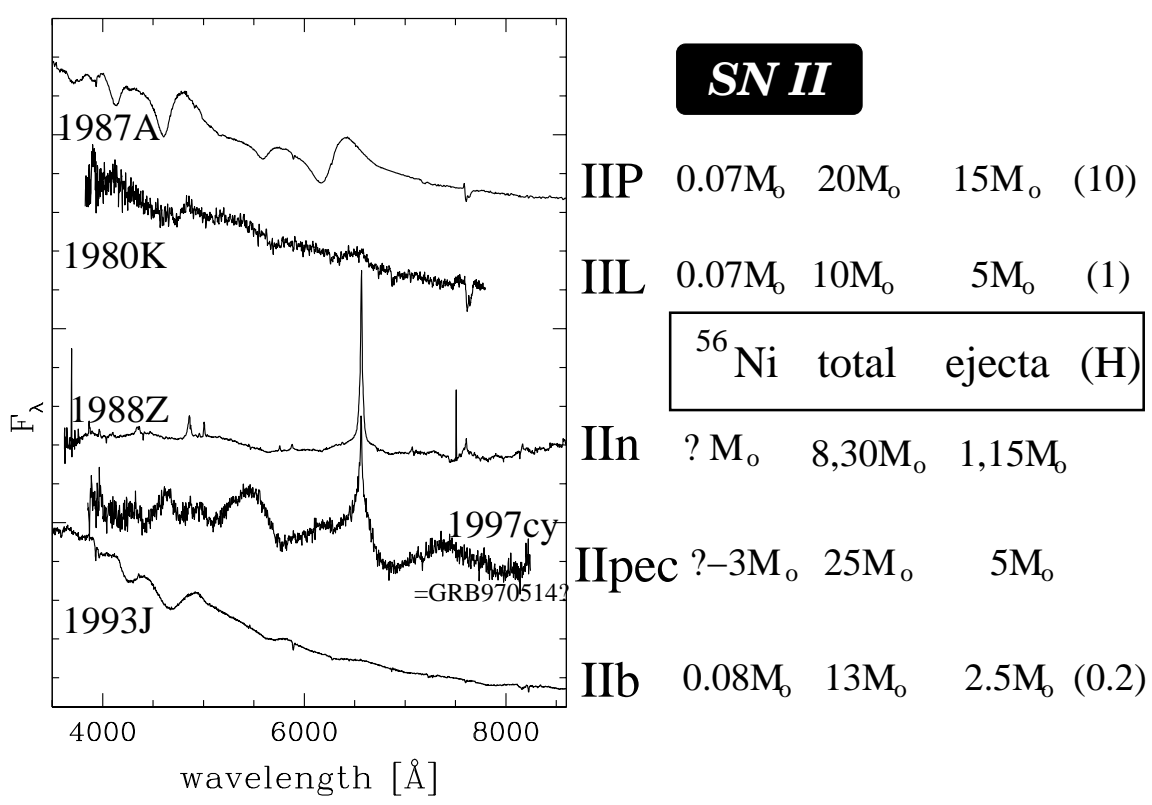

Figure \%. Representative spectra of SN II. On the right we report for each object the best estimate of the ${ }^{56} \mathrm{Ni}$, total and ejecta masses (in parenthesis is the $\mathrm{H}$ mass in the ejecta).

Instead the early luminosity depends on the radius of the progenitor star at the time of explosion. If the progenitor was compact, $10^{12} \mathrm{~cm}$ in the case of SN 1987A, much of the available energy is dissipated for the expansion and until the radioactive decay input becomes dominant, the luminosity remains lower than in the case of an extended progenitor.

In most cases, the remnant of the explosion is expected to be a neutron stars, but it has been argued that the unusual properties of SN 1997D may be understood if the collapsed remnant is a black hole (Turatto et al. 1998).

In general, because of the very high expansion velocity, the emission lines in the SN spectra are very broad but for possible contamination of interstellar gas emissions. A significant fraction of SN II $(10-15 \%$ of the SN II currently discovered) show narrow components on top of broader emissions (Fig. 7). These SNe are labeled IIn, where "n" stands for narrow-line (Schlegel 1990). It turns out that in many cases SN IIn are very bright and their luminosity evolution is much slower than other SN II (Fig. 8). Therefore, an additional source of energy powering the 


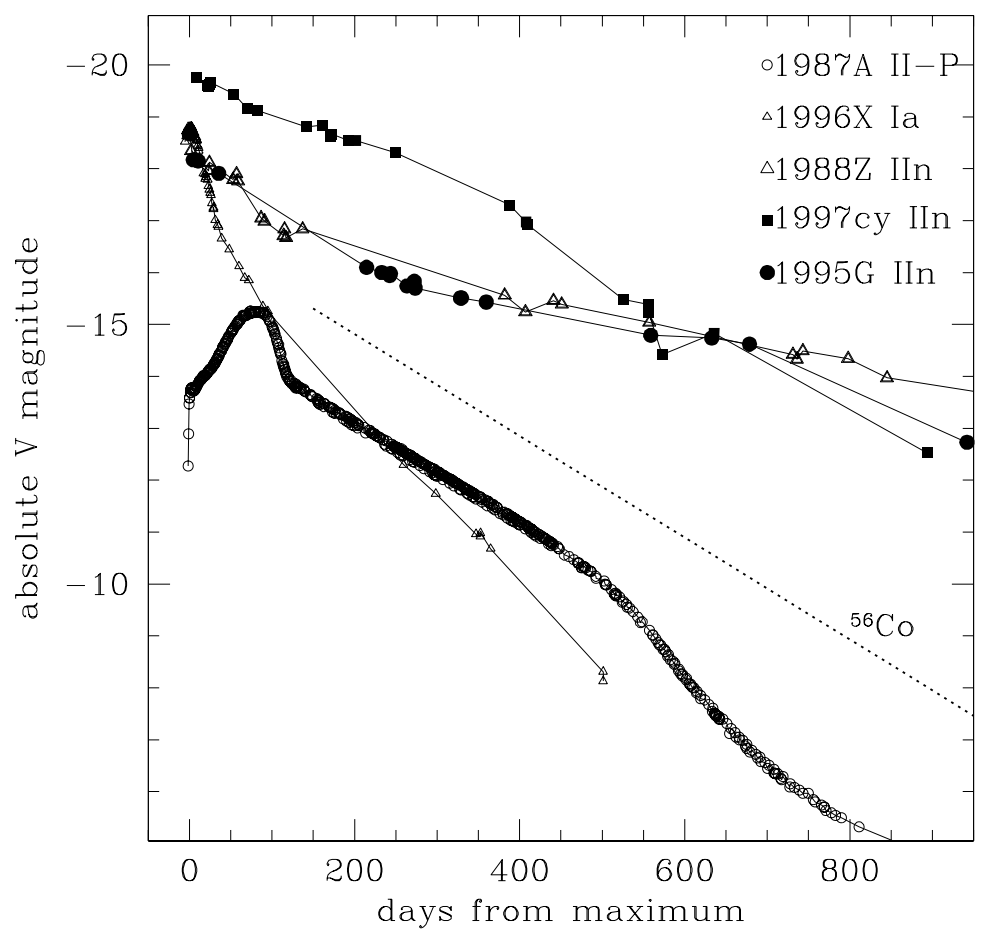

Figure 8. Representative light curves of SN IIn are compared with that of a SN II-P and of a SN Ia. The dotted line is as in Fig. 6

light curve in addition to the radioactive decay is required. It is currently believed that the kinetic energy of the ejecta is converted into radiation when the ejecta shock a dense circumstellar medium (CSM) (Aretxaga et al. 1999). This dense CSM is most likely a relic of a strong mass loss episodes occurring shortly before explosion. Differences in density and distribution of the CSM might explain the large variance of luminosity evolution between different SN IIn.

A most interesting case is that of SN 1997cy for its possible association with GRB970514. Because of the emission line profile (Fig. 7), we believe that also in this case the CSM-ejecta interaction is powering the light curve (Fig.8) and a huge Ni mass is not required (Turatto et al. 2000). 


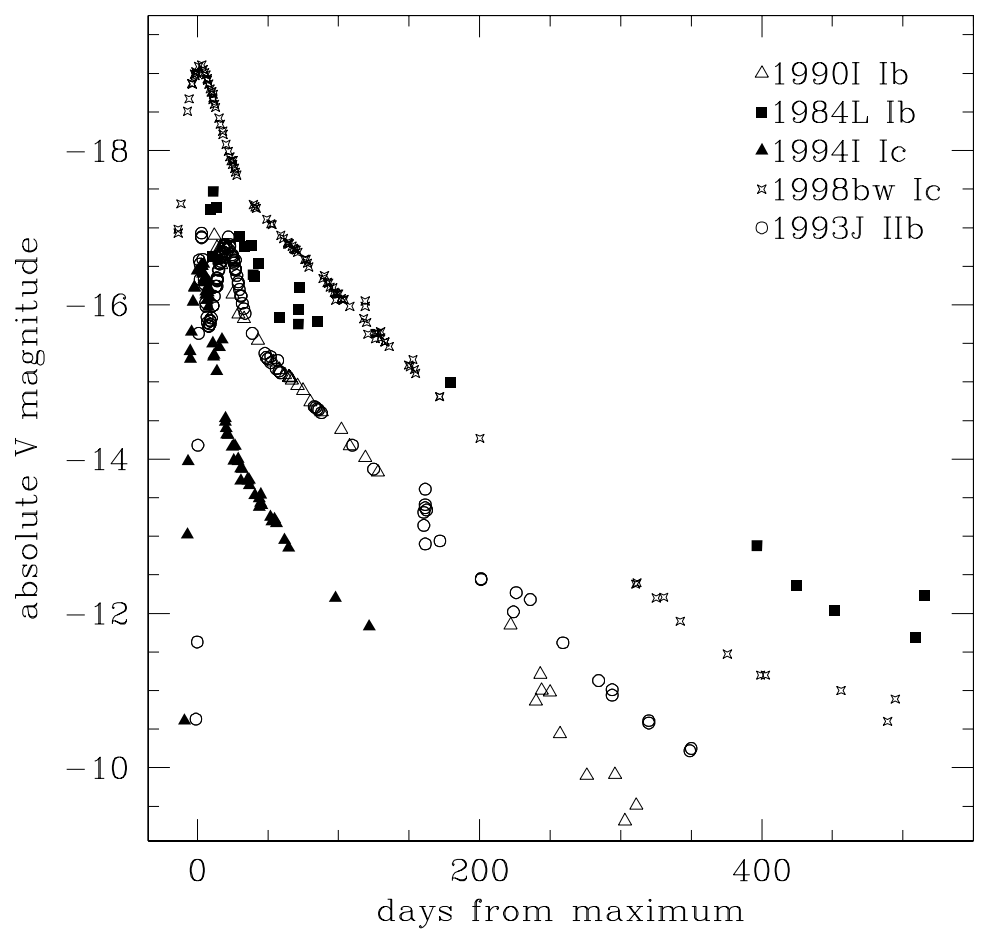

Figure 9. Representative light curves of SN Ib/c.

\section{TYPE IB/C SNE}

The explosion of SN 1993J in M81 was the missing link between SNe of type II and Ib/c. The spectrum of this SN evolved from that of a rather normal type II to that of a type Ib in few weeks. The object is therefore classified as the prototype of the intermediate class of SN IIb. Its observed properties have been explained with the explosion of a massive progenitor with a very small $\mathrm{H}$ envelope $\left(0.2 \mathrm{M}_{\odot}\right)$. It is believed that if the exploding star was left without the $\mathrm{H}$ envelope, the SN would appear as a typical SNIb. If even the He shell was removed the result will closely match the observational properties of type Ic. Other cases of SN Ic in which is detected a small amounts of $\mathrm{H}$ in the ejecta are reported in the literature (cf. Filippenko 1992; 2000H, Pastorello et al. 2000). There are also intermediate cases between type Ib and Ic in which residual amounts of He have been detected (eg. Filippenko et 


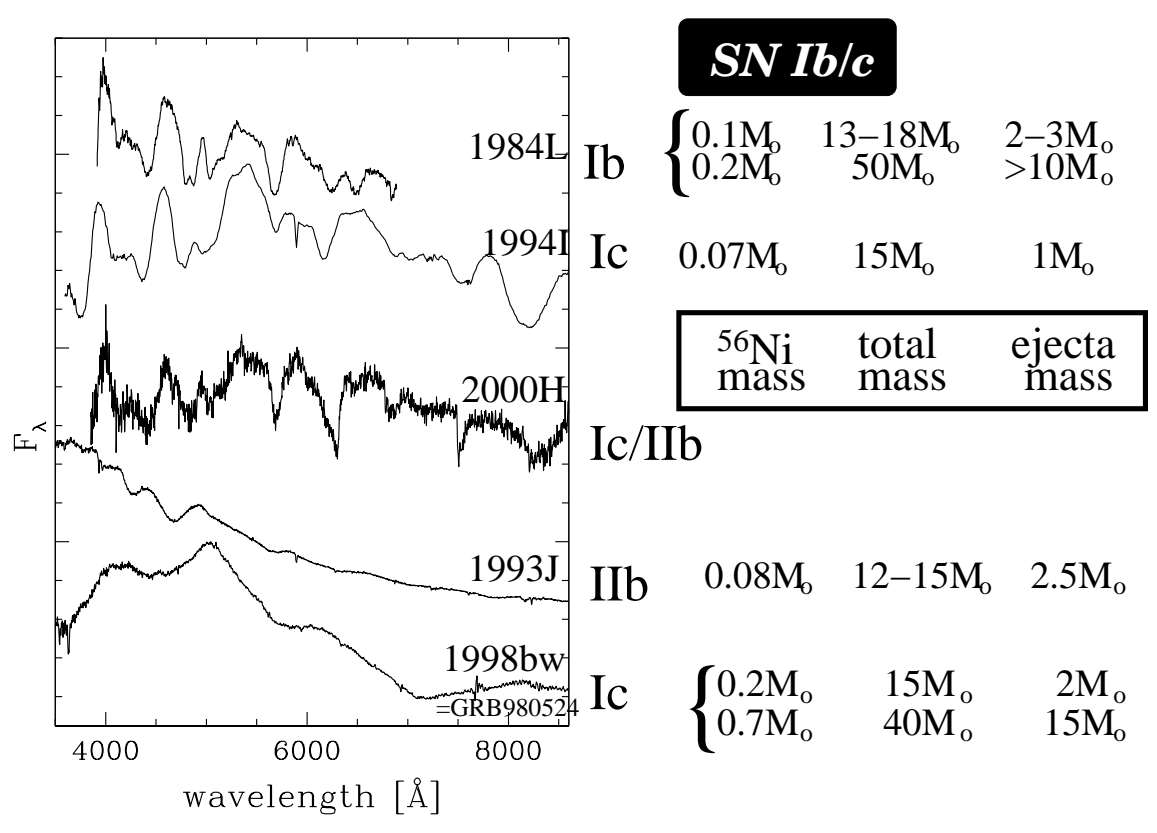

Figure 10. Representative spectra of SNIb/c. On the right the best estimates of the ${ }^{56} \mathrm{Ni}$, total and ejecta masses are reported. In some case different modeling produces significantly different results.

al. 1995). These considerations have motivated the introduction of a unifying scenario where the sequence Ic-Ib-IIb-IIL has mass loss as the driving parameter (Nomoto et al. 1996). At the moment the mechanism for this huge mass loss has not yet been identified, although interaction in close binary systems is the first candidate.

One additional complication is that there are $\mathrm{SN} \mathrm{Ib/c} \mathrm{in} \mathrm{which} \mathrm{the} \mathrm{late}$ light curves seem to track the radioactive decay of ${ }^{56} \mathrm{Co}$ (eg. SN 1984L) and other showing a luminosity decline similar or even faster than that of SN Ia (Fig. 9). Because in all cases it is believed that radioactive ${ }^{56} \mathrm{Co}$ is present, one must admit that the trapping of the $\gamma$-rays from ${ }^{56} \mathrm{Co}$ is different which in turn call for a much smaller ejecta mass in fast declining $\mathrm{SNIb} / \mathrm{c}$ than in the slow declining ones. A summary of the ${ }^{56} \mathrm{Ni}$ and ejecta masses for some $\mathrm{SNIb} / \mathrm{c}$ is reported in Fig. 10

More recently two unusual SNe were discovered (SN 1997ef and 1998bw) that, although formally classified as type Ic because they miss the 6150 $\AA$ dip of SNIa, the $\mathrm{H}$ line of type II and the He lines of type Ib, show indeed a very peculiar spectrum (Fig. 10). This is the result of an ex- 
tremely high expansion velocities and in turn, of high explosion energy (Iwamoto et al. 1998, 2000). Most interesting is the case of SN 1998bw because of his close association with GRB250498 (Patat et al. 2000) Because of these extreme characteristics these SNe, along with SN 1997cy mentioned in the previous section, are often referred as hypernovae and may indeed require specific progenitor scenarios and explosion mechanism.

\section{SN RATES}

The most recent estimate of the rates of the different SN types has been published in Cappellaro et al. (1999) and are summarized in Tab. 1. In average, these values are in fair agreement with previous estimates (cf. van den Bergh \& Tammann 1991).

Table 1. The local SN rates. Units are $S N u=\mathrm{SN} / 10^{10} \mathrm{~L}_{\odot, \mathrm{B}} / 100 \mathrm{yr} . \mathrm{h}=\mathrm{H}_{0} / 100$.

\begin{tabular}{lcccc}
\hline \multirow{2}{*}{$\begin{array}{l}\text { galaxy } \\
\text { type }\end{array}$} & Ia & Ib/c & II & All \\
\cline { 2 - 5 } & \multicolumn{4}{c}{ SN type } \\
\hline E-S0 & $0.32 \pm .11 h^{2}$ & $<0.02 h^{2}$ & $<0.04 h^{2}$ & $0.32 \pm .11 h^{2}$ \\
S0a-Sb & $0.32 \pm .12 h^{2}$ & $0.20 \pm .11 h^{2}$ & $0.75 \pm .34 h^{2}$ & $1.28 \pm .37 h^{2}$ \\
Sbc-Sd & $0.37 \pm .14 h^{2}$ & $0.25 \pm .12 h^{2}$ & $1.53 \pm .62 h^{2}$ & $2.15 \pm .66 h^{2}$ \\
& & & & \\
All & $0.36 \pm .11 h^{2}$ & $0.14 \pm .07 h^{2}$ & $0.71 \pm .34 h^{2}$ & $1.21 \pm .36 h^{2}$ \\
\hline
\end{tabular}

One interesting result is that the rate of SN Ia per unit B luminosity is constant from ellipticals to late spirals. Even if one may argue about what is the stellar population which dominates the blue luminosity in spirals, a significant fraction must derive from young stars. This immediately implies that the average age of the SN Ia progenitors is shorter in spirals than in ellipticals (cf. Fig.5). Also worth noticing is that the $\mathrm{SN} \mathrm{Ib/c} \mathrm{are} \mathrm{only} 15 \%$ of all core collapse events.

Based on the values of Tab. 1, we can derive an estimate of the SN rate in our own Galaxy making the basic assumption that the Galaxy has an average SN rate for his morphological type (here assumed Sb-Sbc) and luminosity $\left(2.3 \times 10^{10} L_{B, \odot}\right)$. If we adopt $\mathrm{H}_{0}=65 \mathrm{~km} \mathrm{~s}^{-1} \mathrm{Mpc}^{-1}$, we expect $0.4 \pm 0.2 \mathrm{SN}$ Ia and $1.5 \pm 1.0 \mathrm{SNII}+\mathrm{Ib} / \mathrm{c}$ per century, that is roughly one SN every 50 years. Formally, this is a factor two lower than derived from counts of historical SNe and SN remnants (eg. van den Bergh \& Tammann 1991, Strom 1994). When considering the large uncertainties however, the two values are not in disagreement. 
For what concerns the SN rate in the local Universe, we have now reached the point where the statistics is no longer limited by the SN discoveries but by the sample of galaxies for which the necessary information (distance, luminosity, morphological types and inclination) are available. In other words, to improve significantly the statistical accuracy of the results, one needs not only to continue SN searches but also to obtain more complete galaxy catalogues.

A major effort is still required, instead, in "un-biased" SN search at high redshift. In fact, the evolution of the SN rate with redshift brings unique information on the progenitor scenarios, galaxy star formation history and initial mass function (eg. Yungelson \& Livio 2000, Dahlèn \& Fransson, C. 1999).

Table 2. Estimate of $\mathrm{SN}$ rates at different redshift. $h=H / 100$

\begin{tabular}{lcccl}
\hline$<z\rangle$ & $\begin{array}{c}\text { Ia } \\
h^{2} \mathrm{SNu}\end{array}$ & $\begin{array}{c}\text { II } \\
h^{2} \mathrm{SNu}\end{array}$ & $\mathrm{N}(\mathrm{SN})$ & source \\
& & & \\
\hline 0.01 & $0.36 \pm 0.10$ & $0.85 \pm 0.41$ & 137 & Cappellaro et al. 1999 \\
0.1 & $0.44_{-0.21}^{+.0 .35}$ & & 4 & hardin \\
0.38 & $0.82_{-0.45}^{+.0 .65}$ & & 3 & Pain et al. 1996 \\
0.55 & $0.62 \pm 0.19$ & & 38 & Pain et al. 2000
\end{tabular}

\# Not yet published. Reported in Ruiz-Lapuente \& Canal 2000.

Current high-z SN searches are aimed to the discovery of SN Ia for their use as distance indicators. By construction, they are not well suited for estimating SN rates. This is obvious from Tab. 2 where we list all observational estimates of SN rates available at "high" redshift and compare them with the local estimates. In this table $\mathrm{N}(\mathrm{SN})$ gives the statistics of SNe on which the estimate is based. The very low statistics and the fact that there is not an estimate yet for core-collapse SN rate outside the local Universe is a clear indication of the work to be done.

\section{References}

Altavilla, G., 2000, Thesis Universita' di Padova.

Aretxaga I., Benetti S., Terlevich R. J., Fabian A. C., Cappellaro E., Turatto M., della Valle M., 1999, MNRAS, 309, 343

Barbon R., Ciatti F., Rosino L., 1979, A\&A, 72, 287

Barbon R., Buondí V., Cappellaro E., Turatto M., 1999, A\&A Suppl., 139, 531

Cappellaro E., Mazzali P. A., Benetti S., Danziger I. J., Turatto M., della Valle M., Patat F., 1997, A\&A, 328, 203

Cappellaro E., Evans R., Turatto M., 1999, A\&A, 351, 459

Clocchiatti A., Benetti S., Wheeler J. C., et al., 1996, AJ, 111, 1286 
Dahlèn, T., Fransson, C.: 1999 A\&A350 359.

Filippenko A. V., 1992, ApJL, 384, L37

Fillipenko A. V., 1997, ARA\&A, 35, 309

Filippenko A. V., Barth A. J., Matheson T., et al., 1995, ApJL, 450, L11

Gaskell C. M., Cappellaro E., Dinerstein H. L., Garnett D. R., Harkness R. P., Wheeler J. C., 1986, ApJL, 306, L77

Hamuy, M., Trager, S. C., Pinto, P. A., Phillips, M. M., Schommer, R. A., Ivanov, V. \& Suntzeff, N. B. 2000, AJ, 120, 1479

Hardin, D., Afonso, C., Alard, C. et al, 2000 A\&Ain press (astro-ph 0006424)

Iwamoto K., Mazzali P. A., Nomoto K., et al., 1998, Nature, 395, 672

Iwamoto K., Nakamura T., Nomoto K., et al., 2000, ApJ, 534, 660

Leibundgut B., Tammann G. A., 1990, A\&A, 230, 81

Mazzali, P.A., Nomoto, K., Cappellaro, E., Nakamura, Y., Umeda, H., Iwamoto, K., 2000, ApJin press (astro-ph/0009490)

Minkowski R., 1941, PASP, 53, 224

Nomoto K., Thielemann F. -., Yokoi K., 1984, ApJ, 286, 644

Nomoto K., Iwamoto K., Suzuki T., Pols O. R., Yamaoka H., Hashimoto M., Hoflich P., van den Heuvel E. P. J., 1996, IAU Symp. 165: Compact Stars in Binaries, 165, 119

Pain R., Hook I. M., Deustua S., et al., 1996, ApJ, 473, 356

Nugent P., Phillips M., Baron E., Branch D., Hauschildt P., 1995, ApJL, 455, L147

Pastorello, A., Altavilla, G., Cappellaro, E., Turatto, M., Benetti S., 2000. IAU Circular 7367.

Patat F., Barbon R., Cappellaro E., Turatto M., 1994, A\&A, 282, 731

Patat, F., Cappellaro, E., Mazzali, P.A., et al., 2000, ApJin press

Perlmutter S., Aldering G., Goldhaber G., et al., 1999, ApJ, 517, 565

Phillips M. M., Lira P., Suntzeff N. B., Schommer R. A., Hamuy M., Maza J. ;, 1999, AJ, 118, 1766

Riess A. G., Filippenko A. V., Challis P., et al., 1998, AJ, 116, 1009

Ruiz-Lapuente, P., Canal, R., 2000, ApJLsubmitted (astro-ph 0009312)

Schmidt B. P., Kirshner R. P., Eastman R. G., et al., 1994, AJ, 107, 1444

Schlegel E. M., 1990, MNRAS, 244, 269

Strom R. G., 1994, A\&A, 288, L1

Turatto M., Bouchet P., Cappellaro E., Danziger, I. J., della Valle, M., Frabsson, C., Gouiffes, C., Lucy, L., Mazzali, P., Phillips, M., 1990, The Messenger, 60, 15

Turatto M., Cappellaro E., Barbon R., della Valle M., Ortolani S., Rosino L., 1990, AJ, 100, 771

Turatto M., Mazzali P. A., Young T. R., et al., 1998, ApJL, 498, L129

Turatto M., Suzuki T., Mazzali P. A., et al., 2000, ApJL, 534, L57

van den Bergh, S. \& Pazder, J. 1992, ApJ, 390, 34

van den Bergh S., Tammann G. A., 1991, ARA\&A, 29, 363

Wheeler, J. C., Benetti, S., in Allen's astrophysical quantities, 4th ed. Publisher: New York: AIP Press; Springer, 2000.̇ं. N. Cox.ed. p. 451.

Woosley S. E., Weaver T. A., 1986, ARA\&A, 24, 205

Woosley S. E., Pinto P. A., Ensman L., 1988, ApJ, 324, 466

Yungelson, L., Livio, M.: 2000 ApJ528 108. 